\title{
Increasing the Efficiency of Milling Stainless Steels by Using a Modified Lubricant-Cooling Fluid
}

\author{
Vladimir Skakun $^{1 *}$ and Eldar Vaniev ${ }^{1}$ \\ ${ }^{1}$ Crimean Engineering and Pedagogical University named after Fevzi Yakubov, Uchebniy side st.8, \\ 295015 Simferopol, Russian Federation
}

\begin{abstract}
This paper considers the possibility of increasing the efficiency of the blade processing process by reducing the cutting forces during end milling by using a modified cutting fluid, which is based on a watermiscible cutting fluid. The conditions for the effective use of modified coolant in the processing of stainless steels were determined, as a result of which there is a decrease in cutting forces, and therefore a decrease in vibrations, which contributes to an increase in the quality of manufactured products. A decrease in cutting forces is associated with the presence in the modified cutting fluid of oleic acid containing surfactants, which form a dense lubricating film on the surface of the cutting tool. When mixing oleic acid in a water-miscible cutting fluid, a special soap solution is additionally used, which significantly improves the solubility, however, over time, oleic acid is separated from the total volume and tends to the surface. To ensure the homogeneity of the medium, a special device has been developed that allows continuous mixing of the compositions, due to the presence of impellers with a differently oriented arrangement of blades. To save lubricant, in the process of research, the technology of minimum quantity lubrication (MQL) was applied, using the Noga Minicool device, which allows portionwise (dosed) supply of lubricant to the cutting zone.
\end{abstract}

\section{Problem statement}

In present-day mechanical engineering, various cutting coolant types are used in metal processing by cutting, the variety of which is increasing year by year. The impact of cutting coolants on cutting is mainly determined by the properties of the processed and tool materials, the nature and way of feeding, cutting modes, the processing type, and the tool design and geometric parameters.

The application areas of the process agents intended to intensify cutting, the mechanism of their impact on the process, and creating new more effective cutting coolants have been considered in the studies by V.N. Latyshev, M.I. Klushin, P.A. Rebinder, V.N. Poduraev, L.V. Khudobin, A.S. Vereshchaka, E.G. Berdichevsky, M.B. Gordon, F. Bowden, D. Tabor, et al.

\footnotetext{
* Corresponding author: vladimir.skakun.92@list.ru
} 
It should be noted that a large percentage of austenitic steel parts are manufactured by intermittent cutting, to which milling is mainly related.

\section{Materials and methods of research}

During the cutting, end milling of thin-walled stainless steel products generates vibrations that negatively affect the finished product quality. This is caused by the formation and propagation of microcracks during processing, which can lead to breakage in the course of the part operation. Turbine blades operating under dynamic loads and vibrations are an example of such products. The specifics of processing stainless steels are poor thermal conductivity of this material, due to which temperature concentrates in the cutting tool; therefore, when choosing a cutting coolant, considerable attention should be paid to its cooling properties [1-7]. The study is aimed at improving the lubricating effect while maintaining other positive qualities of the water-miscible cutting coolant, which may contribute to reducing cutting forces, and, consequently, shock loads and vibrations arising during the cutting.

As a result, the existing (standard) cutting coolant has been improved by mixing with oleic acid, which, in turn, contains a surfactant [8-11]. It should be noted that according to [8-11], vegetable oil-based process lubricants have an increased lubricity. The increased lubricating properties of vegetable oils are explained by surfactants contained in their compositions. Surfactant molecules contain hydrophobes and hydrophiles, which are collected on the cutting tool surface like magnets, forming a dense lubricating film, in contrast to mineral oil molecules containing only hydrophobes randomly distributed on the tool surface that explains the lack of dense lubricating film and the lower lubricating effect compared to vegetable oils (Fig. 1).

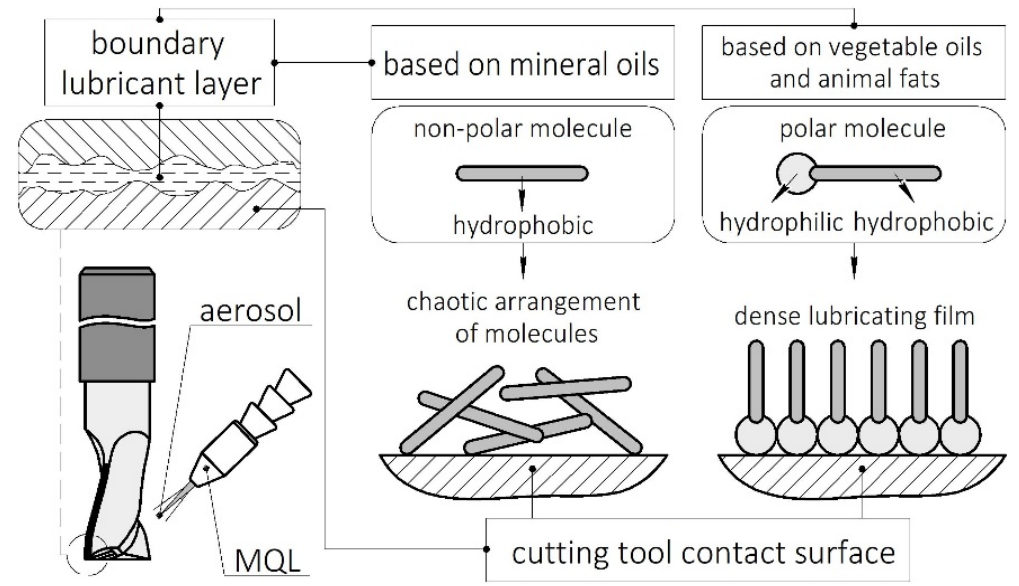

Fig. 1. Schematic arrangement of polar vegetable oil molecules that form a dense lubricating film on the surface of a cutting tool.

All cutting coolants can be classified into four types: solid, plastic, liquid, and gaseous.

The most common are liquid cutting coolants, which are divided into oil and water (water-miscible) classes. Oil-based oils consist of a base mineral oil mixed with antifriction, anti-wear, and extreme pressure additives, corrosion inhibitors, and antioxidants. In such cutting coolants, the mineral oil percentage is $60 \ldots 95 \%$ wt. Usually, these are highly refined naphthenic or paraffinic oils, and a mix of several mineral oils or low-viscosity selectively refined extracts is used as a base for oil cutting coolants. When 
choosing base mineral oils, their physical and chemical properties (viscosity, viscosity index, hydrocarbon-type content) and performance characteristics are first considered.

Water-miscible cutting coolants may contain emulsifiers, petroleum oils, water, alcohols, glycols, corrosion inhibitors, bactericides, anti-wear, extreme pressure, and antifoam additives, electrolytes, and other organic and inorganic products. The advantages of such cutting coolants are higher cooling power, relatively low cost, fire safety, and less toxicity compared to the oil-based ones, and their drawbacks are relatively low lubricating properties, low efficiency in individual operations, and low stability of properties over time.

The cooling effect of cutting coolants is ensured by the heat removal from the heated tool, workpiece, and chip surfaces by convective heat exchange, which inevitably affects the tool life, accuracy, roughness, and residual stress in the surface layer of the part processed and chips. At higher speeds, the temperature of the workpiece being processed is stabilized [3-7].

The Study Objective is to improve the efficiency of using cutting coolants in the end milling of stainless steel $12 \mathrm{X} 18 \mathrm{H} 10 \mathrm{~T}$ (AISI 321 ) by reducing the cutting power parameters.

\section{Statement of the main material}

The experiments involved obtaining data to solve a series of laboratory test problems [1214]. To solve these problems, a test complex has been developed based on a 6P11 vertical mill, meeting the accuracy and rigidity standards according to ГОСТ 7035-75 and ГОСТ 877, which ensures stable mixing of a modified cutting coolant within a single series of experiments. The cutting coolant was fed the cutting zone in an atomized state using the Noga Minicool device designed to implement the minimum lubrication technology (MLT), and the compressed air was supplied using the compressor unit SATVA OL 102. A universal three-component dynamometer M30-3-6k was used to study the cutting force.

To perform experimental studies, the dynamometer with a clamping device and a workpiece was installed on the mill table. The dynamometer was connected through an interface to a PC installed on a separate table next to the test bench, which was used to determine the power parameters of milling (Fig. 2, 3).

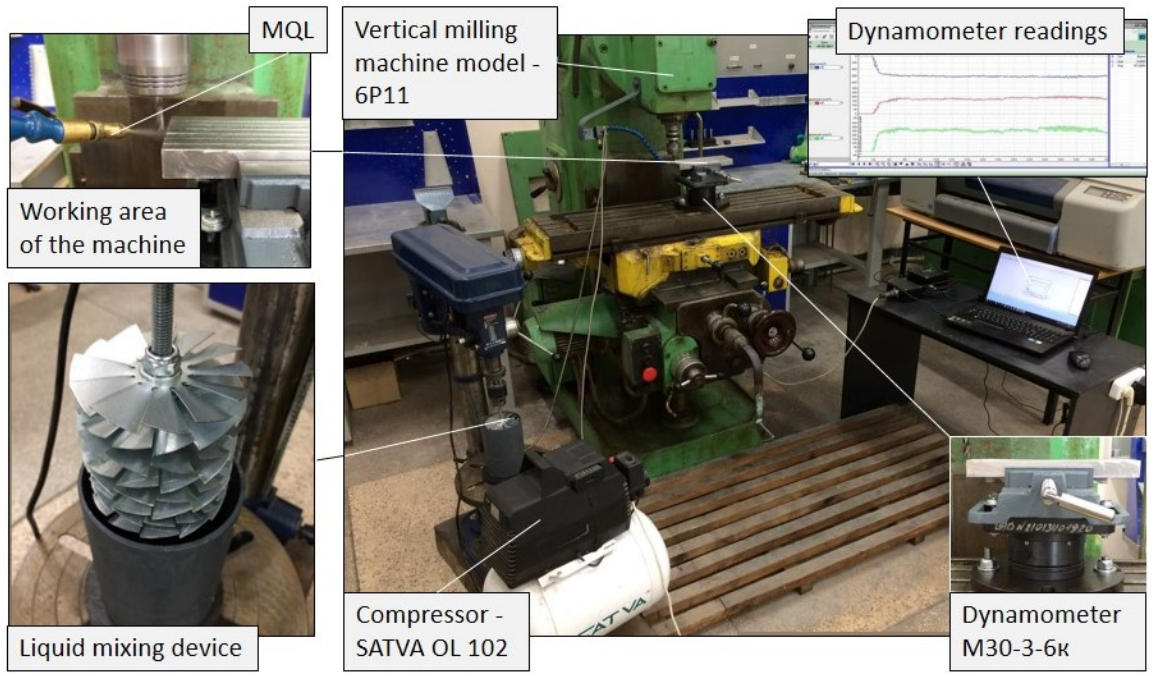

Fig. 2. Experimental bench for measuring the cutting force during milling. 
The scheme for measuring the cutting force components during the end (counter) milling is shown in Fig. 3.

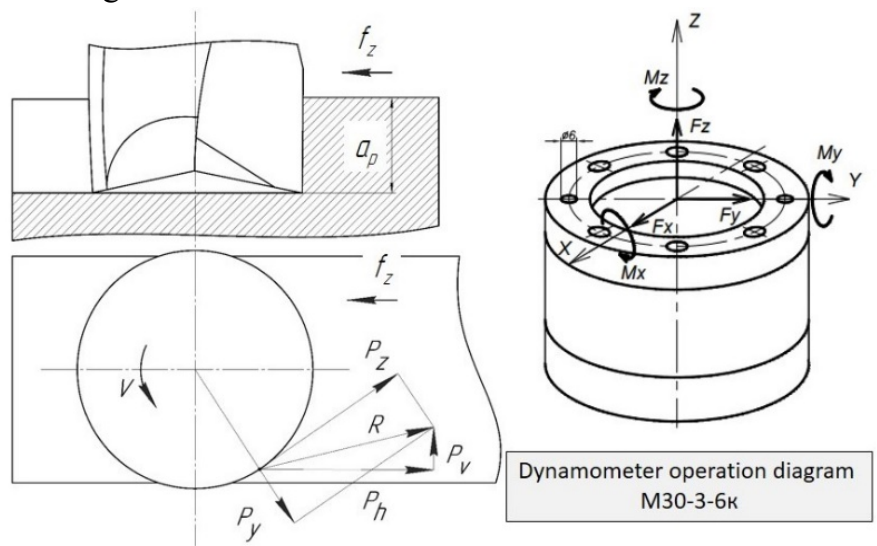

Fig. 3. Cutting force distribution diagram for milling. $f_{z}-$ milling feed; $V-$ cutting speed; $a_{p}-$ depth of cut; $R$ - resulting cutting force.

The cutting coolant was fed between the rear and front surfaces of the cutter teeth. For each experimental point, three parallel experiments were performed, invalid experiments were determined according to the Student's criterion, and when found, the experiment was repeated. To build the experimental data matrices, the average measurement values were taken.

In the course of the experimental studies, one-piece cutters $\varnothing 16 \mathrm{~mm}$ according to ГОСТ 17025-71 were used, made of high-speed steel P6M5 (HSS) of a single batch. Before the tests, the hardness of each tested tooth of the milling cutters was checked (3 times) in the as-received state on the ПМТ-3M device according to the ГОСТ 9450-76 procedure.

At a distance of max $5 \mathrm{~mm}$ from the cutting edges, the cutter body hardness was 63 HRC. The geometric parameters of the end mill and the workpiece processed are shown in Figure 4.

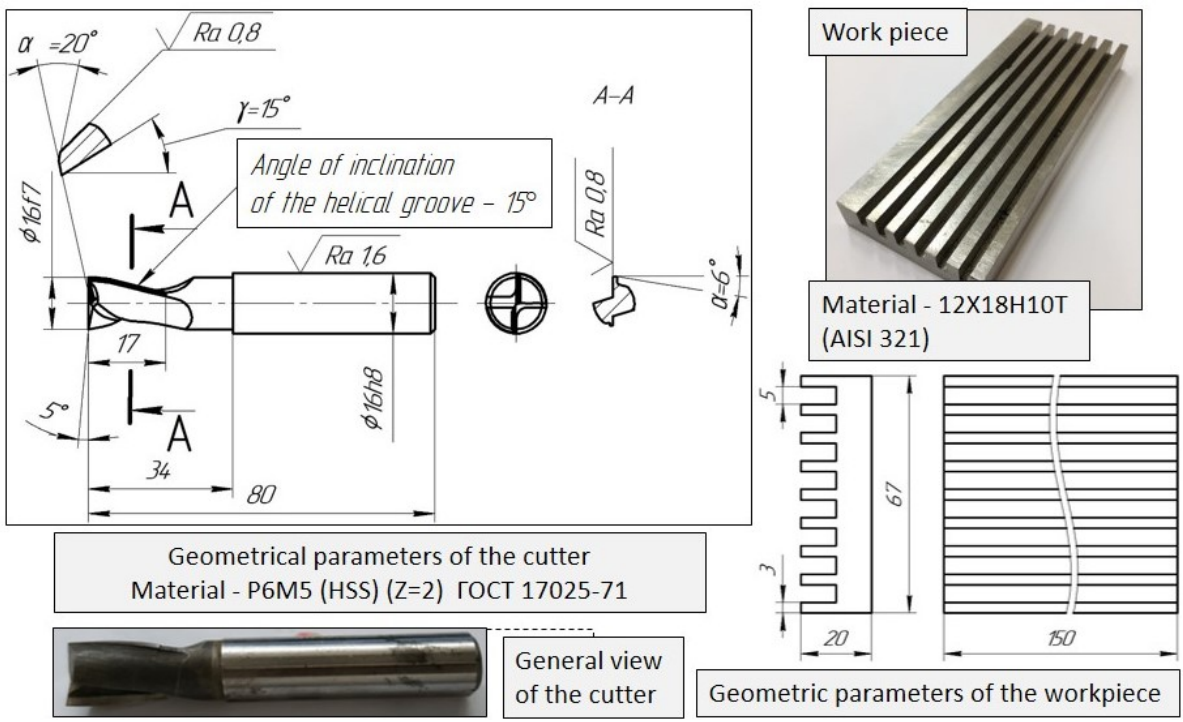

Fig. 4. Geometric parameters cutting tool and workpiece. 
In the experimental studies, a workpiece was used, made of 12X18H10T (AISI 321) stainless steel with ribs that allowed simulating a thin-walled product.

Considering that the modified cutting coolant contains a base in the form of a commercial water-miscible cutting coolant combining detergent, lubricating, and cooling properties, problems have been identified, aimed at modifying the existing commercial cutting coolant by introducing an oleic acid concentrate, which in turn contains a surfactant.

However, due to the different density of oleic acid and water, the problem should be solved, associated with the oleic acid solubility in water, therefore, it has been experimentally revealed that when a soap solution is added to the oleic acid concentrate, the oleic acid solubility in water is significantly improved (Fig. 5), however, as before, the modified cutting coolant requires constant mixing to avoid stratification of fluids, for which a special continuous mixer has been developed (Fig. 2).

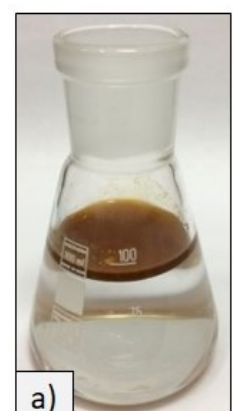

a)

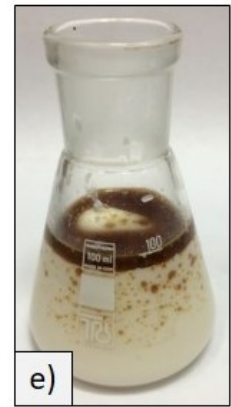

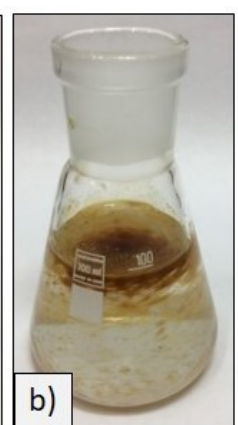

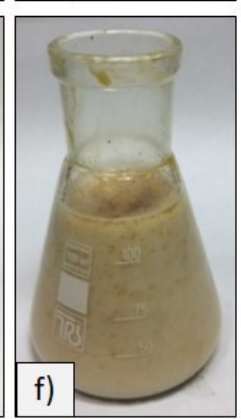

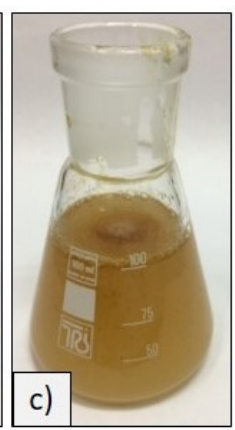

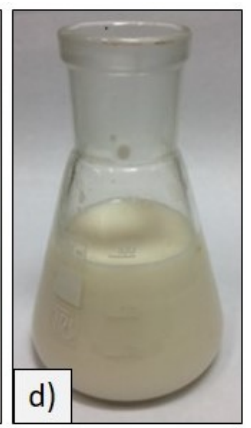

a) - water + oleic acid (before mixing);

b) - water + oleic acid (after mixing);

c) - water + oleic acid + soap solution (after mixing);

d) - emulsion;

e) - emulsion + oleic acid (after mixing);

f) - emulsion + oleic acid + soap solution (after mixing).

Fig. 5. Conditions for obtaining a modified lubricating and cooling technological medium.

The unit for mixing modified cutting coolants comprises a special impeller with differently oriented blades, which allow chaotically mixing the modified cutting coolant, excluding the laminar flow of fluids.

Since only the lubricating property of cutting coolants was investigated, the minimal lubrication technology MLT was used in the study, which allows feeding the lubricant to the cutting zone in portions, doses. Figures 6,7 show graphical dependencies $\mathrm{P}_{\mathrm{h}}=f\left(\mathrm{f}_{\mathrm{z}}\right)$ at a cut depth of $a_{p}=3 \mathrm{~mm}$, respectively, at $\mathrm{V}=10$ and $20 \mathrm{~m} / \mathrm{min}$, and a feed per tooth $\mathrm{f}_{\mathrm{z}}=0.2$ $0.3 \mathrm{~mm} /$ tooth.

The analysis of these dependencies shows that within the given range of the variables, the cutting force component values are determined primarily by the process environment. 


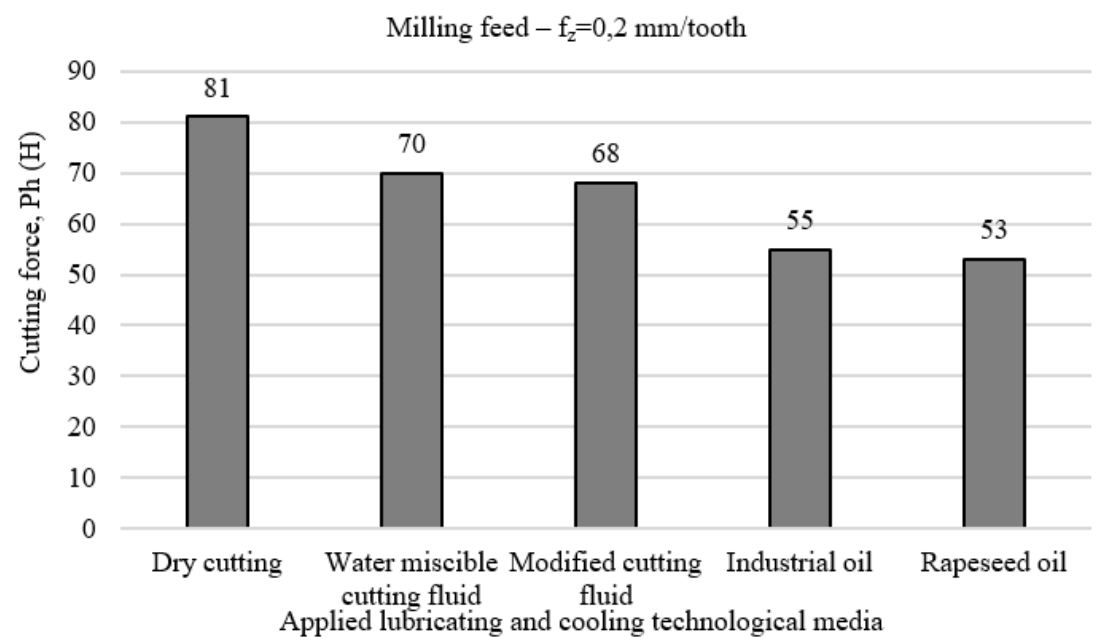

a)

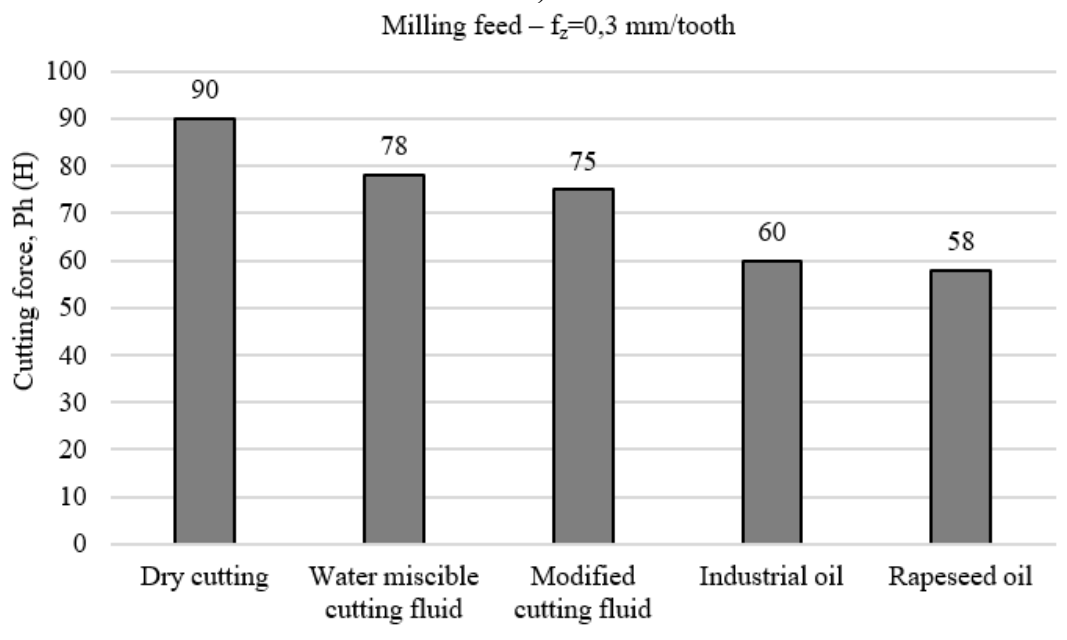

Applied lubricating and cooling technological media

b)

Fig. 6. Changing the cutting force $P_{h}$ in the process of steel (AISI 321) milling, depending on the feed of the workpiece to be processed and the applied cutting fluid.

Cutting parameters in milling: $\mathrm{V}=15 \mathrm{~m} / \mathrm{min} ; \mathrm{a}_{\mathrm{p}}=3 \mathrm{~mm}$. Milling feed: a) $\mathrm{f}_{\mathrm{z}}=0,2 \mathrm{~mm} /$ tooth;

b) $\mathrm{f}_{\mathrm{z}}=0,3 \mathrm{~mm} /$ tooth. LCPM consumption $-15 \mathrm{ml} / \mathrm{h}$. 


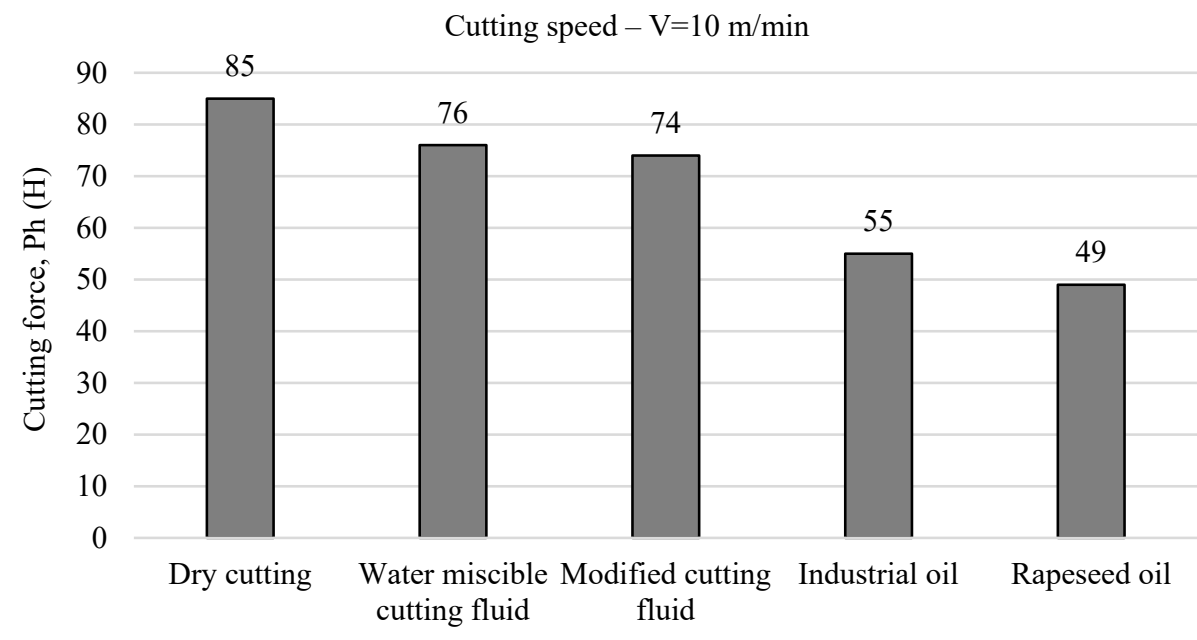

Applied lubricating and cooling technological media

a)

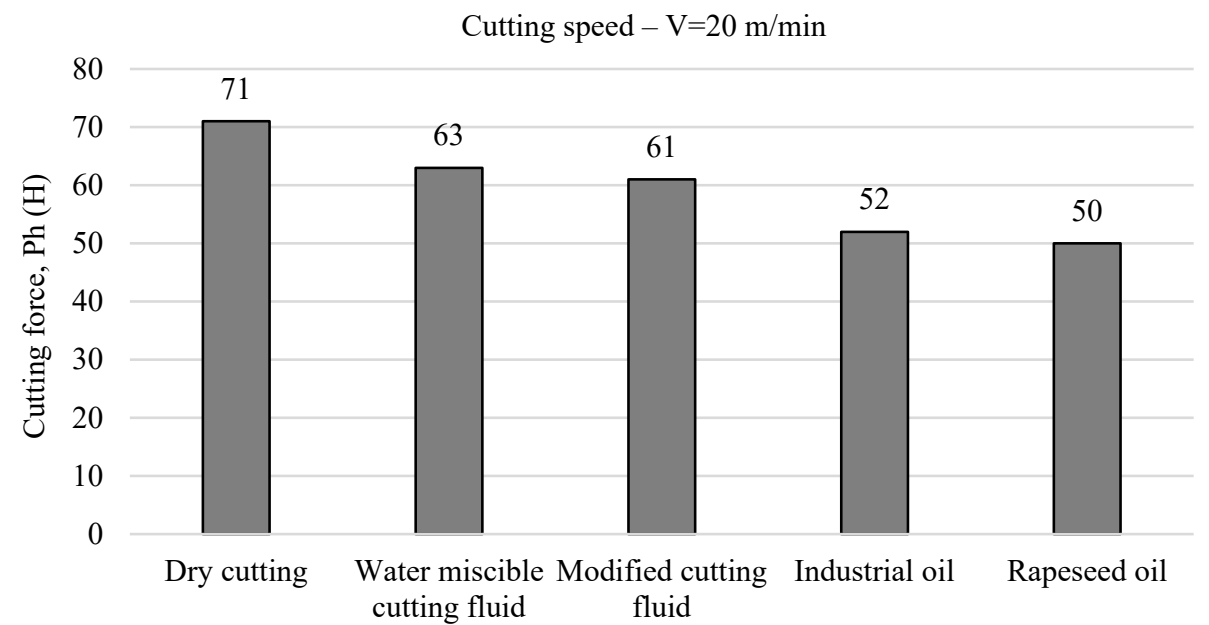

Applied lubricating and cooling technological media

b)

Fig. 7. Changing the cutting force $\mathrm{P}_{\mathrm{h}}$ when milling steel (AISI 321) depending on the cutting speed. Cutting parameters in milling: $f_{z}=0,2 \mathrm{~mm} /$ tooth; $a_{p}=3 \mathrm{~mm}$. Cutting speed: a) $\left.V=10 \mathrm{~m} / \mathrm{min} ; b\right) \mathrm{V}=20$ $\mathrm{m} / \mathrm{min}$. LCPM consumption $-15 \mathrm{ml} / \mathrm{h}$.

The plots show that the modified cutting coolant, having improved lubricating properties compared to the standard water-miscible one, contributes to reducing the force parameters of the $12 \mathrm{X} 18 \mathrm{H} 10 \mathrm{~T}$ (AISI 321) stainless steel milling due to the presence of oleic acid, which has better lubricating properties due to the surfactants in its composition. Projecting the data obtained on milling thin-walled products, e.g., turbine blades made of stainless steels and heat-resistant alloys, and considering the specifics of cutting stainless steels, it can be concluded that an increase in the lubricating properties of a standard water- 
miscible cutting coolant may improve the manufactured product quality by decreasing the cutting force, and therefore, reduce vibrations and shock loads during the cutting, which in turn will reduce the formation and propagation of microcracks while extending the product life.

\section{Conclusions}

The studies have determined the impact degree of the process media and cutting conditions on the power parameters during end milling of 12X18H10T (AISI 321) stainless steel; therefore, a modified water-miscible cutting coolant can be recommended for processing products made of $12 \mathrm{X} 18 \mathrm{H} 10 \mathrm{~T}$ (AISI 321) steel, while the modified cutting coolant combines the advantages of a standard cutting coolant with higher lubricating properties due to the presence of an oleic acid concentrate containing surfactants; a special soap solution, in turn, allows increasing the solubility and obtaining a homogeneous fluid, and high-quality mixing of fluids is ensured by a special device with differently oriented impellers.

Thus, the experimental data obtained on modified cutting coolant in operations proceeding under conditions of pronounced adhesive wear indicate the competitiveness of this coolant compared to the conventional compositions.

\section{References}

1. A.S. Vereshchaka, V.S. Kushner, Cutting materials (Higher school, 2009)

2. V.K. Starkov, Material cutting physics and optimization (Mechanical engineering, 2009)

3. E.G. Berdichevsky, Cooling Lubricants for Material Handling (Mechanical engineering, 1984)

4. D.V. Vinogradov, Application of cooling lubricants for cutting metals (Bauman Moscow State Technical University, 2013)

5. M.I. Klushin, Technological properties of cutting fluid for metal cutting (Mechanical engineering, 1992)

6. D.V. Vinogradov, The main types of cooling lubricants used in cutting metals (Bauman Moscow State Technical University, 2018)

7. L.V. Khudobin, A.P. Babichev, E.M. Bulyzhev and others, Lubricating and cooling technological aids and their use in cutting (Mechanical engineering, 2006)

8. A.I. Aliyev, R.M. Dzhemalyadinov, V.V. Skakun, I.V. Kharchenko, Influence of environmentally friendly technological media on the contact characteristics of the cutting process in the initial period of operation, Bulletin of Rostov State Transport University. Mechanical engineering, v. 1, pp. 8-14 (2019)

9. E.R. Vaniev, V.V. Skakun, R.M. Dzhemalyadinov, Increasing the durability of blade tools with wear-resistant coating by directed action of coolant in the initial period of processing, Scientific notes of the Crimean Engineering and Pedagogical University. Instrumentation, v. 1 (63), pp. 245-251 (2019)

10. V.V. Skakun, R.M. Dzhemalyadinov, R.I. Suleimanov, Experimental evaluation of the influence of vegetable and animal oils on contact processes during turning, Bulletin of the Volgograd State Technical University. Volgograd State Technical University, v. 1 (236), pp. 46-49 (2020) 
11. Ch.F. Yakubov, LCTM strengthening action at metal cutting (Simferopol city printing house, 2008)

12. A.D. Loktev, I.F. Gushchin, V.A. Batuev, and other, General machine-building standards for cutting conditions: / Handbook: Volume 1. (Mechanical engineering, 1991)

13. A.S. Vasiliev, Handbook of a mechanical engineer (Innovative mechanical engineering, 2018)

14. N.I. Sidnyaev, N.T. Vilisova, Introduction to experiment planning theory (Bauman Moscow State Technical University, 2011) 